\title{
EL USO PIONERO DE LA MOSTAZA NITROGENADA POR EMILIO RODA PÉREZ EN EL TRATAMIENTO DE LAS UVEÍTIS IDIOPÁTICAS
}

\author{
BERGUA A $^{1}$
}

\section{INTRODUCCIÓN}

En 1860 el químico inglés Frederic Guthrie (1833-1886) sintentizó el gas mostaza, un arma de guerra química usada durante la Primera Guerra Mundial en julio de 1917 por los alemanes para impedir el ataque británico cerca de Ypern (Bélgica). El gas mostaza también se le conoce con el nombre de Lost, por los descubridores alemanes (Lommel und Steinkopf) o yperita, por el lugar donde se utilizó por vez primera. En 1919 se observaron -además de los efectos tóxicos sobre la piel y mucosas - una profunda leucopenia y aplasia del tejido linfoide en las personas expuestas al gas.

Con un medicamento derivado del gas mostaza desarrollado en la década de los años 20 y 30 del siglo XX, la mostaza nitrogenada, enfermos afectados de linfomas se trataron por primera vez con éxito en la Universidad de Yale (USA) en 1943. La mostaza nitrogenada fue el primero de los llamados agentes alquilantes, compuestos capaces de reemplazar un átomo de hidrógeno en otra molécula por un radical alquilo. Son capaces de combinarse muy activamente con los grupos amino o sulfidrilo de las proteínas y los ácidos nucleicos, causando profundas alteraciones en el código genético de las células expuestas al medicamento, inhibiendo fácilmente su mitosis. La mostaza nitrogenada conserva las propiedades altamente reactivas e irritantes de piel y mucosas del gas del que fue sintetizado y por tanto no puede ser administrada por vía oral sino por vía intravenosa en forma de bolo o en infusión continua.

Su uso terapéutico en oftalmología lo introdujo un internista español, el Dr. Emilio Roda Pérez (1907-1983) en 1950, un hecho apenas conocido de la historia de la medicina y oftalmología española.

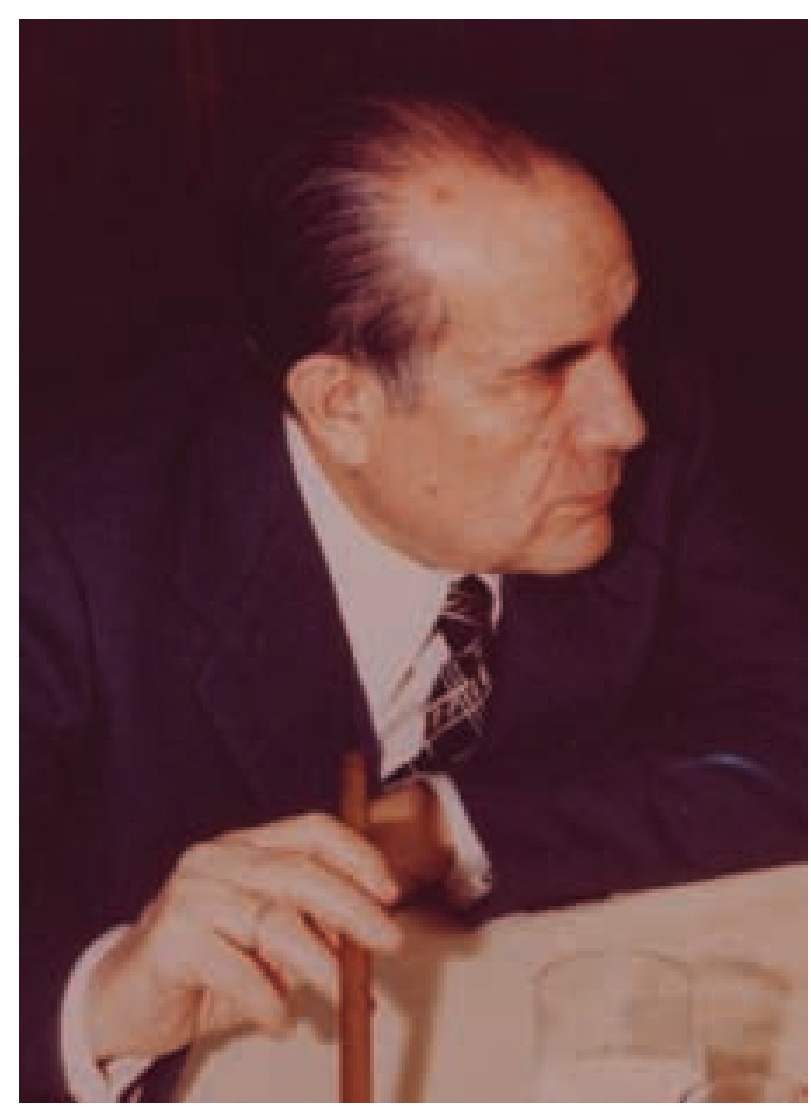

Fig. 1: Fotografía de Emilio Roda Pérez durante un banquete de la Sociedad Española de Medicina Interna.

La mostaza nitrogenada ya no se emplea en oftalmología, pero todavía se sigue utilizando en oncología con el nombre de Mustargen ${ }^{\circledR}$ (Merck, USA), un agente quimioterapéutico conocido también con el nombre de mecloretamina o HN2 hidroclorido. Uno de los primeros países del mundo en usar la mostaza nitrogenada fue España, de la mano del profesor Carlos Jiménez Díaz (1898-1967), que lo

\footnotetext{
1 Doctor en Medicina. Departamento de Oftalmología de la Universidad de Erlangen-Nürnberg. Erlangen. Alemania.

E-mail: antonio.bergua@augen.imed.uni-erlangen.de
} 
utilizó fundamentalmente en el tratamiento de la psoriasis y de la artritis reumatoidea $(3,4)$.

Si bien la mostaza nitrogenada ha sido substituída por otras substancias inmunosupresoras para el tratamiento de las uveítis, un derivado de ella - la ciclofosfamida - todavía forma parte del vademecum oftalmológico. La ciclofosfamida (Endoxan ${ }^{\circledR}$ ) así como la ifosfamida $\left(\operatorname{Holoxan}^{\circledR}\right)$ se derivan de la mostaza nitrogenada y son substancias también alquilantes desarrolladas por el químico alemán Norbert Brock (nacido en 1912) mientras trabajaba para la compañía ASTA (ahora conocida con el nombre de Baxter Oncology) en 1958. Brock y sus colaboradores convirtieron la mostaza nitrogenada en una sustancia menos tóxica, una pro-droga que se transportaba activamente en el interior de las células tumorales. Una vez en ellas esta pro-droga se transforma enzimáticamente en su forma activa, tóxica para la célula, especialmente de los linfocitos (1). Norbert Brock y su equipo sintetizaron más de 1.000 componentes del grupo de la oxazofosforina, entre ellos la ciclofosfamida (2).

El principal uso terapéutico de la ciclofosfamida es el tratamiento de los linfomas, así como algunas formas de leucemia y algunos tumores sólidos, en general combinándola con otras formas de quimioterapia. También se utiliza en el tratamiento de enfermedades autoinmunes, en las que otras «disease-modifying antirheumatic drugs» (DMARDs) se muestran inefectivas. Especialmente eficaz con ciclofosfamida es el tratamiento de la glomerulonefritis asociada al lupus eritematodes sistémico.

En oftalmología el uso de la ciclofosfamida se limita a aquellos procesos inflamatorios no infecciosos en los que otros compuestos inmunosupresores se muestran inefectivos o se requiere una potencia inmunosupresora mayor que la que se lograría con substancias convencionales. Entre estas indicaciones destacan las vasculitis producidas por la enfermedad de Wegener (5), la enfermedad de Behçet (6), la oftalmía simpática (7), etc.

\section{UN INTERNISTA PIONERO EN EL TRATAMIENTO SISTÉMICO DE LAS INFLAMACIONES INTRAOCULARES}

Emilio Roda Pérez nació en febrero de 1907 en Cuenca. Se licenció en Madrid en junio de 1931.
Doctor en Medicina por la Universidad Nacional Mayor de San Marcos, Lima (Perú), una universidad pública fundada en 1551, la más antigua de América, en octubre de 1958. Hizo investigación en Würzburg (Alemania) desde 1933 a 1936, con el Profesor Tanhauser, sobre el metabolismo de los fosfolípidos en el hígado de la rata. Posteriormente a la Guerra Civil española trabajó hasta el año 1963 en Hospital Clínico de San Carlos de Madrid en el Servicio de Medicina Interna del Profesor Jiménez Díaz. Entre los años 1959 a 1961 estuvo activo profesionalmente como «Visiting Professor in Pathology» en la Louisiana State University en Nueva Orleans (USA). Llegó a publicar entre 80 y 100 trabajos en diferentes revistas médicas, pero más frecuentemente en La Revista Clínica Española. Falleció en Madrid el 21 de marzo de 1983 (datos facilitados por su hijo, el neurocirujano madrileño Dr. Enrique Roda Frade).

\section{EL USO DE LA MOSTAZA NITROGENEDA EN LAS UVEÍTIS DE TRATAMIENTO REFRACTARIO}

El primer trabajo sobre el uso de la mostaza nitrogenada en el tratamiento de las inflamaciones intraoculares lo presentó Emilio Roda en el Congreso Internacional de Reumatismo, celebrado en Barcelona el 24 de septiembre de 1951 y posteriormente en el XXIX Congreso de la Sociedad Oftalmológica Hispanoamericana, que se celebró en Madrid entre el 1 y el 5 de octubre del mismo año (8). Fruto de sus experiencias clínicas con la mostaza nitrogenada Roda publicó tres trabajos entre 1951 y 1953 (9-11). Trató un total de seis pacientes, de los que cinco pudo documentar extensamente y uno interrumpió el tratamiento. De los cinco pacientes tratados cuatro evolucionaron positivamente y en uno, «por cierto excepcionalmente avanzado», no obtuvo una mejoría clínica significativa.

El primer paciente tratado con mostaza nitrogenada fue una mujer madrileña peluquera de 33 años de edad atendida por vez primera por Roda Pérez el 20 de noviembre de 1950 (8). Padecía desde hace quince años una uveítis en el ojo derecho de origen desconocido. Las reacciones de Wassermann eran negativas, así como las tuberculínicas. El ojo izquierdo se afectó de forma transitoria, con dismi- 
nución de la visión y la presencia de dolores retrooculares «muy intensos», siete años atrás. Pero siete meses antes de consultar al Dr. Roda Pérez remitida por el Dr. Manuel López Enríquez- el ojo izquierdo empeoró de nuevo. El ojo derecho, ya ciego desde hace tiempo, también se vio afectado de dolores muy intensos, lo que aconsejó su enucleación, que realizó el doctor Hermenegildo Arruga (1886-1972). Esta paciente fue tratada desde el comienzo de su uveítis en 1936 con todos los tratamientos existentes en aquella época como eran la fototerapia, salicilatos, midriáticos, antiluéticos (¿sales de mercurio?), tuberculina, etc. En 1944 fue tratada cuando empeoró el ojo izquierdo con 20 gramos de estreptomicina, sin obtener mejoría alguna. Una leve mejoría - aunque transitoria - se consiguió tras una aplicación subconjuntival e intramuscular de 600.000 Unidades de Penicilina. «Fue entonces» — señala Roda Pérez- «y por las razones que después señalaremos, cuando aconsejamos el tratamiento de esta paciente con mostaza nitrogenada, que por primera vez que sepamos, después de una búsqueda minuciosa de la literatura mundial, se realizaba en este tipo de procesos. Se le administraron cuatro inyecciones en los días 24 y 27 de noviembre y 1 y 16 de diciembre del año 1950 . La dosis administrada en cada inyección fue de $5 \mathrm{mg}$.; $20 \mathrm{mg}$ en total». Las inyecciones fueron aplicadas por el Doctor Carrión. No se produjeron en la paciente así tratada reacciones secundarias inmediatas, ni en el lugar de la inyección ni de forma generalizada. El hemograma se mantuvo normal en cuanto a los hematíes, pero se produjo una disminución continuada de los leucocitos. Por este motivo como tratamiento coadyuvante se le administraron a la paciente una dieta hiperproteica rica en vitaminas del grupo $\mathrm{B}$ y vitamina $\mathrm{C}$ así como un preparado a base de pentanucleótido. La última exploración ocular, que se realizó en la clínica del Doctor Carreras, ponía de manifiesto la presencia de Tyndall en la cámara anterior y sinequia posterior en el sector inferior de la pupila. En el fondo de ojo se apreciaba algún foco coroideo «evolucionado», pero no pigmentado.

En el segundo trabajo publicado sobre el tratamiento de las uveítis idiopáticas con mostaza nitrogenada, Roda Pérez informa sobre cuatro casos más, además de resumir de nuevo la evolución clínica del señalado anteriormente (9). El segundo paciente tratado con mostaza nitrogenada era una joven madrileña de veinte años de edad. Dos años antes de ser tratada presentó un cuadro de disminución transitoria de la visión en ambos ojos así como empezó a notar «nieblas» en el campo visual del ojo izquierdo. Las pupilas se dilataron y deformaron. Pérdida de apetito y adelgazamiento de nueve kilos en dos meses. Una exploración oftalmológica más detenida mostró «una marcada inyección ciliar y conjuntival» en los dos ojos. Además, el ojo izquierdo presentaba un iris inflamado, sinequias posteriores en forma de hoja de trébol. También se observó una turbidez difusa del vítreo con flóculos flotantes. El ojo derecho presentaba signos de inflamación mucho menos acusados. El tratamiento con inyecciones intravenosas de mostaza nitrogenada, $5 \mathrm{mg}$ cada semana, produjo en el ojo izquierdo «una acusada mejoría de su agudeza visual, habiendo desaparecido la inflamación del iris con reabsorción de los exudados de vítreo». En el ojo derecho la turbidez del vítreo desapareció completamente. Esta paciente mantuvo una mejoría permanente desde el tratamiento con mostaza nitrogenada. El tercer paciente fue una mujer zaragozana de 38 años de edad afectada en el ojo derecho de una «iritis difusa metastásica, de probable origen reumático». El tratamiento con la misma pauta de mostaza nitrogenada lo tolera de forma perfecta. La paciente fue controlada desde el punto de vista oftalmológico por el profesor Tena Ibarra que refiere que después de una semana de tratamiento los fenómenos inflamatarios del ojo derecho habían desaparecido, «no existiendo exudados en la cámara anterior». La cuarta paciente, de 34 años, referida por el profesor Carreras, padecía de una uveítis bilateral, «muy antigua, con oclusión y seclusión pupilar en ojo izquierdo y coloboma quirúrgico de iris a las VIII horas, siendo imposible ver el fondo en este ojo. En el derecho presenta sinequia posterior casi total, excepto a las VIII horas. El fondo se ve muy dificilmente a causa de la turbidez del vítreo y cuerpos flotantes del mismo». El tratamiento con mostaza nitrogenada se mostró inefectivo. La quinta paciente, de 47 años, padeció una enucleación del ojo derecho a los 20 años de edad después de perder la vista a causa de las complicaciones producidas por unas «ulceraciones corneales» después de padecer el sarampión cuando tenía siete años. El ojo izquierdo, normal hasta un mes antes de referir la paciente al Dr. Roda Pérez, padeció «repentinamente de moscas volantes y fosfenos», notando una acusada pérdida de visión acompañada de cefaleas gravitativas muy 
intensas. La exploración oftalmoscópica del único ojo mostraba una inyección ciliar y conjuntival muy marcada así como una pupila irregular por la presencia de sinequias múltiples. El fondo de ojo no era visible debido a la turbidez del vítreo. Después de un mes de tratamiento con mostaza nitrogenada el fondo de ojo era de nuevo visible, ya que los exudados de la cámara anterior y la turbidez del vítreo habían remitido. Los fénomenos inflamatorios del iris habían desaparecido, con persistencia de las sinequias posteriores. «La enferma subjetivamente se encuentra extraordinariamente mejorada y dice ver infinitamente mejor que lo hacía cuando empezó su tratamiento». Una sexta y última enferma, de diecisiete años fue también tratada por una uveítis anterior idiopática pero sin terminar el protocolo establecido por el Dr. Roda ya que la paciente no acudió más a la consulta.

En el tercer y último trabajo (9) se relatan las mismas experiencias clínicas y terapéuticas que en el aparecido en La Revista Clínica Española en 1951. Pero en esta ocasión la discusión es más amplia que en los trabajos anteriores así como, al final de la exposición de los casos, aparecen una serie de comentarios de mano de Costi, Mario Esteban, Gustavo Leoz de la Fuente, y López Enríquez. El Doctor Costi justifica el empleo existoso de la mostaza nitrogenada «por su interferencia en el sistema de reacción». Refiere, además, que tiene una experiencia de tres pacientes con iritis idiopática que trató con mostaza nitrogenada conjuntamente con el Dr. Parra. «Los resultados fueron excelentes en los tres, desapareciendo los signos bioquímicos de inflamación de iris». Y más adelante añade: «El mayor inconveniente de esta técnica es que el oftalmólogo no siempre la conoce lo suficiente para emplearla directamente; pero esto se puede vencer enviando los enfermos al internista para su tratamiento, igual que hacemos con los diabéticos, nefríticos, hipertensos, etc.».

Para Mario Esteban (que fue uno de los oftalmólogos que exploró los pacientes tratados por el Doctor Roda) «los problemas que plantean las uveítis hay que enfocarles fundamentalmente desde el punto de vista etiopatogénico, base para establecer una terapéutica racional». .../... «Desgraciadamente, vemos uveítis que ni por caracteres clínicos ni por las comprobaciones de laboratorio acertamos a calificar» .../... «A la mostaza nitrogenada no podemos concederle la virtud de actuar etiológicamente, sino sólo la de modificar los tejidos y la actividad celular en un sentido más o menos favorable» .../... «Y por lo que respecta a la mostaza nitrogenada, como medicación empleada en otras afecciones, por ejemplo, en el Hodking, sus efectos inmediatos son a veces magníficos, pero poco duraderos, y su aplicación es delicada y no exenta de cuidados».

El doctor Gustavo Leoz de la Fuente conocía los casos tratados con mostaza nitrogenada, que fueron seguidos por el doctor Bartalozzi en la clínica del profesor Carreras, donde trabajaban. «Efectivamente», comenta Leoz de la Fuente, «el tratamiento etiológico es el ideal, y con toda seguridad el más eficaz. Ahora bien, en muchas iritis, iridociclitis y uveítis la etiología, a pesar de todas las tentativas, permanece desconocida, y en estos casos es donde conviene no empeñarse en hacer un tratamiento etiológico, esperando sin hacer nada, o muy poco, y repitiendo las investigaciones y exploraciones clínicas. Es como al que se le quema la casa y, antes de llamar a los bomberos, empieza por buscar el origen del fuego, y cuando llegan aquéllos, ya no tienen nada que apagar porque está todo quemado. La mostaza nitrogenada nos permite, con los cuidados necesarios, hacer algo, y generalmente eficaz, cuando no tenemos nada que hacer».

Por útimo, López Enríquez estimó «de gran interés deslindar las indicaciones de las mostazas nitrogenadas en oftalmología y, muy principalmente, comparar su valor curativo en relación con otros medios, ya clásicos, de aplicación más sencilla».

El reconocimiento a la labor pionera en el tratamiento de las uveítis por medio del empleo de la mostaza nitrogenada del Emilio Roda Pérez no ha pasado desapercibida en la literatura anglosajona. Así, en una introducción histórica sobre el empleo farmacológico de la ciclofosfamida (su variante menos tóxica) en oftalmología, Albert Vitale y Stephen Foster citan los trabajos pioneros del internista español (11).

\section{BIBLIOGRAFÍA}

1. Zhang J, Tian Q, Yung Chan S, Chuen Li S, Zhou S, Duan $W$, et al. Metabolism and transport of oxazaphosphorines and the clinical implications. Drug Metab Rev 2005; 37: 611-703.

2. Brock N. The history of the oxazaphosphorine cytostatics. Cancer 1996; 78: 542-547.

3. Jimenez Diaz C, Lopez Garcia, Merchante A, Perianes J. Treatment of rheumatoid arthritis with nitrogen mustard; preliminary report. J Am Med Assoc 1951; 147: 14181419. 
4. Jiménez Díaz C, Merchante A, Perianes J, López García E. Treatment of psoriasis with nitrogen mustard. Rev Clin Esp 1950; 39: 341.

5. Damico FM, Kiss S, Young LH. Sympathetic ophthalmia. Semin Ophthalmol 2005; 20: 191-197.

6. Evereklioglu C. Current concepts in the etiology and treatment of Behcet disease. Surv Ophthalmol 2005; 50: 297350 .

7. Wardyn KA, Ycinska K, Matuszkiewicz-Rowinska J, Chipczynska M. Pseudotumour orbitae as the initial manifestation in Wegener's granulomatosis in a 7-year-old girl. Clin Rheumatol 2003; 22: 472-474.
8. Roda Pérez E. Sobre un caso de uveítis de etiología ignota tratado con mostaza nitrogenada. Rev Clin Esp 1951, 41: 265-267.

9. Roda Pérez E. El tratamiento de las uveítis de etiología ignota con mostaza nitrogenada. Rev Clin Esp 1952; 44. 173-180.

10. Roda Pérez E. El tratamiento de las uveítis de etiología ignota con mostaza nitrogenada. Archivos Soc Oftalmol Hisp Am 1952; 12: 131-151.

11. Vitale AT, Foster CS. Immunosuppressive chemotherapy. In: Zimmerman TJ. Textbook of Ocular Pharmacology. Philadelphia: Lippincott-Raven; 1997; I: 723-761. 